\title{
Neural Network Application for Netted Radar Information Processing Systems
}

\author{
Hu Jian Hu Fanjun \\ Department of Aerial Ammunition Engineering \\ The First Aeronautics College of Air Force \\ Xinyang, Henan, China \\ E-mail:13523896492@163.com
}

\begin{abstract}
This paper discusses the neural network application for the information processing in the netted radar tracking systems compared with the problems of the conventional radar information processing. And then test the neural network using simulation method. The simulation result shows that the neural network method can perfectly solve the target tracking problems in the netted radar systems.
\end{abstract}

Keywords-application; neural network; information processing; radar tracking systems

\section{INTRODUCTION}

With the development of the air traffic controlling system and the military defense system, the requirement of the target tracks, particularly the high maneuvering target tracks, is higher and higher. On the other hand, the electronic countermeasures, including the anti-radiation missile and anti-stealth technique, threaten the air defense systems, particularly the radar tracking systems. Therefore, it is necessary to study the radar information processing.

Reference [1] gives us the advantages and disadvantages of the radar tracking systems. In recent years, with the increasing development of the neural network method, because of its large-scale parallel processing, distributed information memory, better adaptability and self-organizing ability, as well as the learning association, tolerance etc., it is possible for us to give play to the advantages and solve the problem of the radar information processing. Particularly, compared the neural network with the traditional adaptive system, the neural network not only has more powerful learning capability, but also has the memory, selection, abstract and identification abilities. Through learning neural network, it automatically finds the solution to the problem. At the same time, it can be tolerant of the data and environments uncertainties, and particularly suit to the signal detection, parameter estimation, target feature extraction and identification and system model establishment etc.. The application of the neural network to find the solution to the target tracking problems in the radar information processing come into true not far away. This paper discusses the application of the neural network to the information processing in the netted radar tracking systems.

\section{CONVENTIONAL RADAR INFORMATION PROCESSING}

When research the information processing in the netted radar tracking systems, at first, should establish the mathematical model of the target motion [2]. The conventional method is to establish the target motion model in the common coordinate reference system, where the origin is at the main site. At the same time, in the netted radar tracking systems, many plots fall in the same correlation gate; furthermore, the number of the plots correlating with a certain track is usually more one, so the netted radar data correlation is involved. The conventional method is to set up a proper correlation gate, which the success correlation probability is maximum within the limits of the given miscorrelation probability. In addition, since each radar measuring precision is different, hence, its reliability is different too. Moreover, in the netted radar tracking systems, the uncertainties in the communication networks cause additional errors, the multiradar integration algorithm minimizes the effects of communication networks uncertainties in both local estimation and central integration.

As known, on establishing the target motion every radar model, the difference of its geographical position, the target motion regularity is different; the conventional method has certain limitation [3]. When performing the data fusion and the plot-track correlation in the netted radar tracking systems, the traditional method doesn't not only realize the autotracking multitarget, but also needs so much computation that "the fusion explosion" is caused [2,4]. Hence, it is difficult to realize the real-time tracking. In order to solve these problems, the application of the neural network to the radar information processing is a better way.

\section{NEURAL NETWORK INFORMATION PROCESSING}

The application of the neural network to the radar information processing in the netted radar tracking systems, bases on the conventional tracking method by means of the learning function, the large-scale parallel distributed memorizing and processing capabilities, as well as the collective operating capability of the neural network [5], accomplishes the target tracking with precision and solves the problems in the conventional method, such as the model limitation, the fusion explosion, the inference complication. At last, accomplishes the adaptive inference and improve the real-time processing ability and adaptability of the netted radar tracking systems.

The schematic diagram of the application of neural network to the radar information processing in the netted radar tracking system is shown in Fig. 1. 


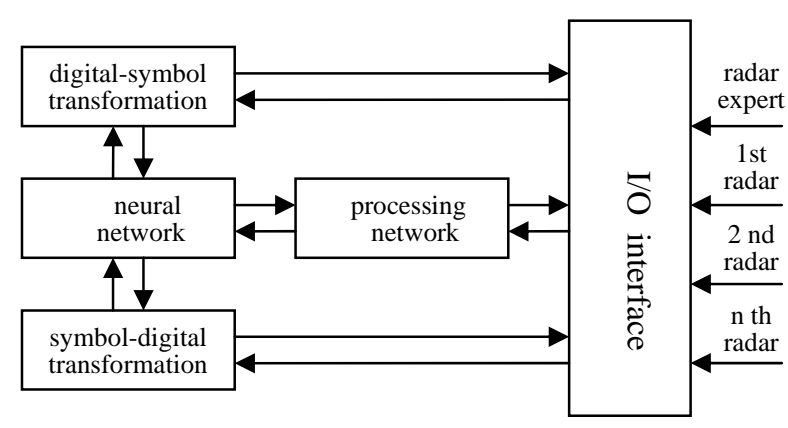

Figure 1. The schematic diagram of the netted radar tracking system

In the neural network netted radar tracking system, at first, should build the neural network model. Secondly, decide the learning algorithm according to the characteristic of the netted radar tracking system. Finally, study the problems relating to the target tracking. At present, there are many neural network models and can select the selforganizing neural network and the multi-level feed forward neural network model. Reference [6] indicates that the Hopfield model and BCS (Boundary Counter System) model can be adopted to perform the multitarget tracking processing. Now, the paper adopts the ART (adaptive Resonance Theory) neural network model in studying the netted radar tracking.

ART neural network model consists of the input and output nerve cells, which computes the matching score, that is, the output values of the output nerve cells, through the feed forward connecting weights and sample input. The nerve cell activity with the largest matching score is enhanced through the lateral inhibiting between the output nerve cells. The other output nerve cells activity degrees are gradually decreased. The feedback connecting from the output nerve cell to the input nerve cell performs learning and comparing.

ART neural network model can be described with the differential equation and perform self-organization with the competitive learning [7].The adjacent processing units restrain one another, finally, only one processing unit is active, this active processing unit represents the present plot of the target.

The architecture of the ART neural network model can be divided into the caring subsystem and adjusting subsystem. By means of the two mutual compensating subsystems and interaction of the controlling mechanism, the target data is obtained. The caring subsystem solves the evaluation of the netted radar target tracking and the configuration performance when it performs in the known environment, for example, the covering area, the tracking quality and the filtering capability. The adjusting subsystem solves the uncertainty and quality evaluation in the netted radar tracking systems, including the radar spatial location, the measuring time alignment, the uncertainties of the coordinate transformation and the communication. The following kind of system is considered.

$$
\begin{gathered}
\mathrm{d} \boldsymbol{X}(\mathrm{t}) / \mathrm{dt}=\boldsymbol{f}(\boldsymbol{X}(\mathrm{t})) \\
\boldsymbol{Z}(k+1)=\boldsymbol{h}(\boldsymbol{X}(k+1))+\boldsymbol{V}(k+1)
\end{gathered}
$$

Where, $\boldsymbol{X}$ is of the $n$ dimensions of the target state vector, $\boldsymbol{Z}$ is of the $\boldsymbol{m}$ dimensions of the measuring vector, $\boldsymbol{V}$ is of the $m$ dimensions of the measuring noise vector, $\boldsymbol{f}(\boldsymbol{X})$ is the function of the $n$ dimensions of the target real value state vector, $\boldsymbol{h}(\boldsymbol{X})$ is the function of the $m$ dimensions of the target real value state vector, $\boldsymbol{X p}$ is target state estimation under the minimum mean square error, let

$$
\begin{gathered}
\boldsymbol{X} \boldsymbol{p}(k / k)=\mathrm{E}(\boldsymbol{X}(k) / \boldsymbol{Z}(k)) \\
\boldsymbol{Z}_{k}=\{\boldsymbol{Z}(\mathrm{j}): \mathrm{j}=1,2, \ldots, \mathrm{k}\} \\
\boldsymbol{X} \boldsymbol{p}(k+1 / k)=\boldsymbol{X} \boldsymbol{p}(k / k)+\boldsymbol{f}(\boldsymbol{X} \boldsymbol{p}(\mathrm{k} / \mathrm{k})) \triangle \mathrm{t} \\
+\boldsymbol{F}\left(\boldsymbol{X} \boldsymbol{p}(k / k) \boldsymbol{f}(\boldsymbol{X} \boldsymbol{p}(k / k)) \triangle \mathrm{t}^{2} / 2\right.
\end{gathered}
$$

$\boldsymbol{X p}(k+1 / k+1)=\boldsymbol{X p}(k+1 / k)+\boldsymbol{K}(k+1)[Z(k+1)-\boldsymbol{h}(\boldsymbol{X} \boldsymbol{p}(k+1 / k))](6)$

$$
\boldsymbol{K}(k+1)=\boldsymbol{P}(k+1 / k) \boldsymbol{H}^{\mathrm{T}}(\boldsymbol{X} \boldsymbol{p}(k+1 / k))[\boldsymbol{H}(\boldsymbol{X} \boldsymbol{p}(k+1 / k))
$$$$
\left.\boldsymbol{P}(k+1 / k) \boldsymbol{H}^{\mathrm{T}}(\boldsymbol{X} \boldsymbol{p}(k+1 / k))+\boldsymbol{R}(k+1)\right]^{-1}
$$$$
\boldsymbol{P}(k+1 / k)=\boldsymbol{\Phi}(k) \boldsymbol{P}(k / k) \boldsymbol{\Phi}^{\mathrm{T}}(k)+\boldsymbol{Q}(k)
$$

$$
\boldsymbol{Q}(k)=\boldsymbol{I}+\boldsymbol{F}(\boldsymbol{X} \boldsymbol{p}(k / k)) \triangle \mathrm{t}
$$

$$
\boldsymbol{P}(k+1 / k+1)=[\boldsymbol{I}-\boldsymbol{K}(k+1) \boldsymbol{H}(\boldsymbol{X} \boldsymbol{p}(k+1 / k))] \boldsymbol{P}(k+1 / k)
$$

$$
\boldsymbol{F}(\boldsymbol{X p}(k / k))=\mathrm{d} f / \mathrm{dt}(X=X \boldsymbol{p}(k / k))
$$$$
\boldsymbol{H}(\boldsymbol{X} \boldsymbol{p}(k+1 / k))=\mathrm{d} \boldsymbol{h} / \mathrm{d} \boldsymbol{X}(\boldsymbol{X}=\boldsymbol{X} \boldsymbol{p}(k+1 / k))
$$

The quality index " $\boldsymbol{X}$ " can be defined as the linear combination with proper weights $\left(W_{1}, W_{2} \cdots\right)$, of partial quality indices $\left(X_{1}, X_{2} \cdots\right)$, each refering to the parameters listed above. The weights are selected in order to emphasis those indices having more operational relevance, i. e., the corresponding activity degrees of the nerve cell are increased. The overall quality index is:

$$
X(k, E)=\sum_{i} W_{i} X_{i}(k, E) / \sum_{i} W_{i}
$$

It is a function of time " $k$ " and the particular operational environment " $\boldsymbol{E}$ " considered.

The quality index assessed so far is compared with the operational requirements expressed in terms of an ideal quality index to be achieved. If the operational requirements are not satisfied to any extent, the manager-controller in the I/O interface adjusts the performance, the data fusion, the transfer of information and control commands and makes proper reconguration to satisfy the requirements.

Because the ART neural network model is the kind of self-organizating neural network model, it forms the response to input through competition. In the application of the neural network to the radar information processing in the netted radar tracking systems, need the pre-processing network that the target motion possibility and the radar performance parameters are described.

The target motion possibility can be described with any probability function [8]. Now, select the Gauss distribution on target range, bearing and maneuvering radius. The radar performance parameters are mainly the radar location, the measurement precision and transformation precision, etc.. The pre-processing network performs some pre-processing in terms of the target motion possibility and the radar performance parameters. For example, the collecting of the target feature data is formed with series connecting the Fourier-Mellin Transformation (FMT) with the Coding Transformation (CT), i. e., the Coding Transformation is 
performed according to the FMT results of the sampling target data. Code the non-negative component with " 1 " and the other with " 0 ", then can obtain a series of the 2-array sequences. The target is characterized by the existing frequencies of the specific double-bit codes and the specific three-bit codes in the sequences. Therefore, in the application of the ART neural network to solving the netted radar target tracking problems, every processing unit represents one point in the feature space. Furthermore, the number of the processing unit denoting the same position is one more. These processing units represent the various target motion direction respectively. The connection of the processing unit is mutual inhibiting connection. At the same time, the present target plots obtained by the netted radar target tracking systems and the all processing units where the area is covered with the Gauss distribution in the target motion direction is mutual stimulated connection, the mutual connecting weights relate to the corresponding Gauss distribution. The ART neural network modifies the weights according to the proper rules and learning algorithms, executes the self-organization through the competitive learning tracking the target.

\section{SIMULATION AND CONCLUSION}

In order to demonstrate the neural network radar information processing method in the netted radar tracking systems, compare the neural network radar information processing results with the conventional radar information processing results. The following conditional parameters are selected. There are three radars in the netted radar tracking systems. The distance between the radars is $150 \mathrm{~km}$. The detection probability has been assumed to be $p_{D}=1$. The neural network radar tracking system solves the problems through selecting one of the eight target motion directions at every moment. The sampling interval $T=0.01 \mathrm{~s}, \omega=0.42 \mathrm{~s}^{-1}$, The measuring noise $R(k)=1.64 * 10^{-4} \quad(\mathrm{rad})^{2}, \Phi$ $=\left[\begin{array}{cc}1 & T \\ -\omega^{2} T & 1\end{array}\right], N=10$. The target tracking state error $\sigma_{X}^{2}(k)$ is the assessment criterion of the target tracking precision [9].

$$
\sigma_{X}^{2}(k)=\frac{1}{N} \sum_{i=k-N+1}^{k}\left[X_{p}(i / i)-X(i)\right]^{2}
$$

The simulation result applying the neural network to three radars tracking systems to track the targets is shown in TABLE I. The simulation result shows that the application of the neural network can solve the target tracking problems in the netted radar tracking systems, such as the "fusion and explosion" and error. At last, the target tracking precision in neural network radar information processing is much better than that in the conventional radar information processing

TABLE I. THE TARGET TRACKING PRECISION COMPARISON

\begin{tabular}{|l|c|c|c|c|c|}
\hline \multicolumn{1}{|c|}{$\boldsymbol{t} / \mathbf{s}$} & $\mathbf{1}$ & $\mathbf{5}$ & $\mathbf{1 0}$ & $\mathbf{1 5}$ & $\mathbf{2 0}$ \\
\hline $\begin{array}{l}\text { The } \\
\text { conven- } \\
\text { tional }\end{array}$ & $\begin{array}{c}3.805^{*} \\
10^{-3}\end{array}$ & $\begin{array}{c}7.625^{*} \\
10^{-2}\end{array}$ & 0.2006 & 0.2509 & 0.3022 \\
\hline $\begin{array}{l}\text { The } \\
\text { neural } \\
\text { network }\end{array}$ & $\begin{array}{c}2.685^{*} \\
10^{-4}\end{array}$ & $\begin{array}{c}1.364^{*} \\
10^{-4}\end{array}$ & $\begin{array}{c}1.012^{*} \\
10^{-3}\end{array}$ & $\begin{array}{c}2.195^{*} \\
10^{-4}\end{array}$ & $\begin{array}{c}2.365^{*} \\
10^{-3}\end{array}$ \\
\hline
\end{tabular}

\section{REFERENCES}

[1] A. Farina, Radar Data Processing, Beijing: National Defense Industry Press, 1992.

[2] HE You, XIU Jianjuan and ZHANG Jingwei, Radar Data Processing with Applications, Beijing: House of Electronics Industry, 2009.

[3] Shalom B, William D B, Multitarget-Multisensor Tracking Applications and Advances, USA: Artech House, 2001.

[4] Bolic M, Djuric P M and Hong S, "Resampling Algorithms and Architectures for Distributed Particle Filters”, IEEE, Transactions on Signal Processing, vol. 53, no. 7, Nov. 2005, pp. 2442-2450.

[5] Shie-Jue Lee, Chun-Liang Hou, “An ART-Based Constrouction of RBF Neural Networks", IEEE Transactions on Neural Networks vol. 13, no. 6, Nov. 2002, pp. 1308-1321.

[6] FENG Ding, Neural Network Expert Systems, Beijing: Science Press, 2006.

[7] ZHANG Laiyao, YAN Pingfan, Neural Network and Fuzzy Control, Beijing: Tsinghua University Press, 1998.

[8] Proakis G, Rader C M, Ling F, et al, Algorithm for statistical Signal Processing, New Jersey, USA:Prentice-Hall, 2002.

[9] CAI Qingyu, XUEyi, Phased Array Radar Data Processing and Its Simulation Techniques, Beijing: National Defense Industry Press, 1997. 